\title{
Fetal left ventricular modified myocardial performance index and renal artery pulsatility index in pregnancies with isolated oligohydramnios before 37 weeks of gestation
}

\author{
(D) Rıza Madazlı1, (D Hakan Erenel ${ }^{1}$, (D) Ayşegül Özel ${ }^{1}$, (D Funda Öztunç² \\ 1Department of Obstetrics and Gynecology, Division of Perinatal Medicine, İstanbul University-Cerrahpaşa, Cerrahpaşa \\ Faculty of Medicine, İstanbul, Turkey \\ 2Department of Pediatrics, Division of Pediatric Cardiology, İstanbul University-Cerrahpaşa, Cerrahpaşa Faculty of Medicine, \\ İstanbul, Turkey
}

\section{Abstract}

Objective: The aim was to evaluate fetal left modified myocardial performance index (Mod-MPI) and renal artery Doppler parameters in fetuses with isolated oligohydramnios and a normal amount of amniotic fluid.

Material and Methods: This was a prospective cohort study; 25 pregnancies with isolated oligohydramnios and 25 healthy, gestational agematched controls, between $24+0$ to $36+6$ weeks of gestation, were recruited. Primary outcome was to compare left modified MPI and mean fetal renal artery pulsatility index (PI). The secondary outcome was to compare adverse perinatal outcomes between the groups.

Results: Mean Mod-MPI was significantly higher $(p=0.001)$ and isovolumetric relaxation time was longer ( $p=0.009)$ in the isolated oligohydramnios group. Mean renal artery PI values were not different between the groups. Birthweight $(p=0.041)$ and gestational age at birth $(p=0.001)$ were significantly lower, and incidences of delivery before 37 weeks $(p=0.034)$ and Cesarean section due to non-reassuring fetal heart rate testing $(\mathrm{p}=0.021)$ were significantly higher in women with isolated oligohydramnios than the control group. We found no significant relationship between Mod-MPI and adverse perinatal outcomes.

Conclusion: Fetuses with isolated oligohydramnios have increased left Mod-MPI, which may be due to mild cardiac diastolic dysfunction. Increased Mod-MPI is not associated with adverse perinatal outcomes and does not seem to help in the management of pregnancies before 37 weeks of gestation with isolated oligohydramnios. (J Turk Ger Gynecol Assoc 2021; 22: 286-92)

Keywords: Isolated oligohydramnios, myocardial performance index, perinatal outcome, preterm, renal artery Doppler

Received: 26 September, 2019 Accepted: 04 June, 2020

\section{Introduction}

Amniotic fluid volume assessment is a standard part of obstetric sonography and abnormalities of the amniotic fluid volume are related to increased risk for adverse perinatal outcomes (1). An amniotic fluid index (AFI) $\leq 5 \mathrm{~cm}$ or a single vertical pocket of amniotic fluid $\leq 2 \mathrm{~cm}$ is the most common definition for oligohydramnios. Approximately $0.5 \%$ to $5 \%$ of pregnancies are complicated by oligohydramnios
(2). Oligohydramnios without fetal structural and chromosomal abnormalities, intrauterine infection, uteroplacental insufficiency, premature rupture of membranes and known maternal disease is known as isolated oligohydramnios (3). According to the literature, obstetrical outcomes of pregnancies with isolated oligohydramnios are controversial. Some studies have shown that isolated oligohydramnios is a predictor of adverse outcome $(4,5)$ while others have not confirmed the association $(3,6)$. 
Fetal cardiac function can be evaluated non-invasively by measuring Doppler-derived myocardial performance index (MPI) (7). Lower variability and higher inter- and intra-observer agreement were obtained with improved modification of myocardial performance index (Mod-MPI) (8). Higher left Mod-MPI values were demonstrated to be associated with left ventricular dysfunction (9). Left Mod-MPI has been investigated in a number of complicated pregnancies to evaluate fetal cardiac dysfunction (10-12). To the best of our knowledge, there is no study focusing on fetal cardiac function in isolated oligohydramnios.

The main component of amniotic fluid is fetal urine in the second and third trimester. Thus, decreased amniotic fluid volume can be seen secondary to impaired fetal renal function (13). Renal arterial perfusion affects urine output directly, which can be measured with renal artery Doppler parameters. However, results of studies focusing on alteration in renal Doppler parameters in isolated oligohydramnios were not in agreement and so it is difficult to state that isolated oligohydramnios is associated with renal artery Doppler parameters (14-16).

The aim of this study was to investigate whether pregnancies before 37 weeks of gestation with isolated oligohydramnios differ in measures of fetal cardiac function and renal artery flow velocity waveforms from those with pregnancies with normal amniotic fluid. The outcome of pregnancies between these two groups was also compared and the value of the left Mod-MPI in the prediction of adverse perinatal outcomes was evaluated.

\section{Material and Methods}

This prospective cohort study was conducted between August 2017 and June 2018 in the İstanbul University unit at Cerrahpaşa, in the Cerrahpaşa Faculty of Medicine, Department of Obstetrics and Gynecology, Division of Perinatology. After ethical approval (İstanbul University-Cerrahpaşa, Cerrahpaşa Faculty of Medicine Ethical Committee (approval number: 215728), twentyfive pregnancies with isolated oligohydramnios between $24+0$ to $36+6$ weeks of gestation were recruited. An AFI $\leq 5 \mathrm{~cm}$ was defined as oligohydramnios. These subjects were matched with 25 healthy, gestational age-matched singleton pregnancies with normal amniotic fluid (AFI: 8-25 $\mathrm{cm}$ ). Exclusion criteria included: multiple pregnancies; preeclampsia; pregestational or gestational diabetes; other chronic diseases; premature rupture of membranes; fetal growth restriction; fetal infection; structural or chromosomal abnormalities; and pregnancies with pathological umbilical and uterine artery Doppler indices. During the study period, Voluson E10 C4-8-D (GE Healthcare, Zipf, Austria) curved and convex array probes were used.
After informed consent was obtained, a detailed fetal anatomy examination was performed and AFI was calculated using the 4-quadrant method (1). In the apical four-chamber view, the Doppler sample gate was kept at $3 \mathrm{~mm}$ and located on the left wall of the ascending aorta, between the leaflets of the aortic valves (AV) and mitral valves (MV). The angle of insonation was between 0 and 300, and the fastest Doppler sweep (15 $\mathrm{cm} / \mathrm{s}$ ) was used. Isovolumetric contraction time (ICT) was measured from MV closure to AV opening. Ejection time (ET) was measured from $\mathrm{AV}$ opening to closure. Isovolumetric relaxation time (IRT) was measured from AV closure to MV opening (Figure 1). The Mod-MPI was calculated using the formula (ICT + IRT)/ET. Three consecutive measurements were performed and the mean value was used for calculation. To evaluate renal artery blood flow, coronal plane was obtained and the Doppler gate was located on the proximal third of the renal arteries (Figure 2). The angle of insonation was close to $0^{\circ}$. Three consecutive measurements were made for the calculation of mean left and right renal artery pulsatility index (PI).

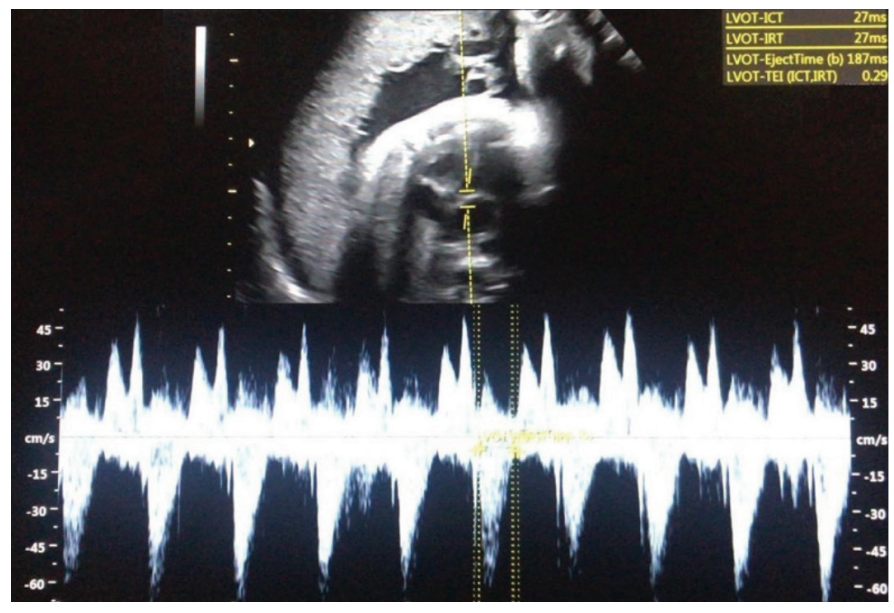

Figure 1. Measurement of fetal left ventricle myocardial performance index

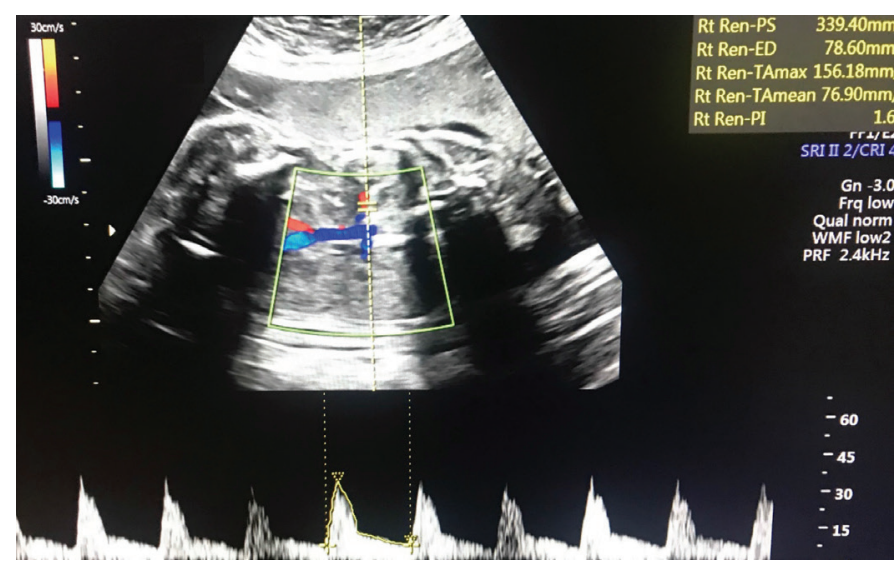

Figure 2. Measurement of fetal renal artery Doppler 
Pregnancies with isolated oligohydramnios were followed every 2-3 weeks with umbilical artery Doppler until 32 weeks of gestation and every 1-2 weeks with non-stress test (NST) and umbilical artery Doppler until 36 weeks of gestation and twice weekly thereafter. Persistently non-reactive NST was used as an indication for delivery. Labor was induced in isolated oligohydramnios cases at 39 weeks gestation if not delivered before. Pregnancies with normal amniotic fluid were followed every four weeks until 36 weeks of gestation and examined at 38 weeks and 39 weeks of gestation and twice weekly thereafter until 41 weeks of gestation. Labor was induced at 41 weeks gestation if not delivered before. The follow-up algorithm for patients is shown in Figure 3. Route of delivery, gestational age at birth, birth weight and the presence of meconium-stained amniotic fluid at delivery were evaluated. Primary outcome was to compare left Mod-MPI and mean fetal renal artery PI and secondary outcome was to compare adverse perinatal

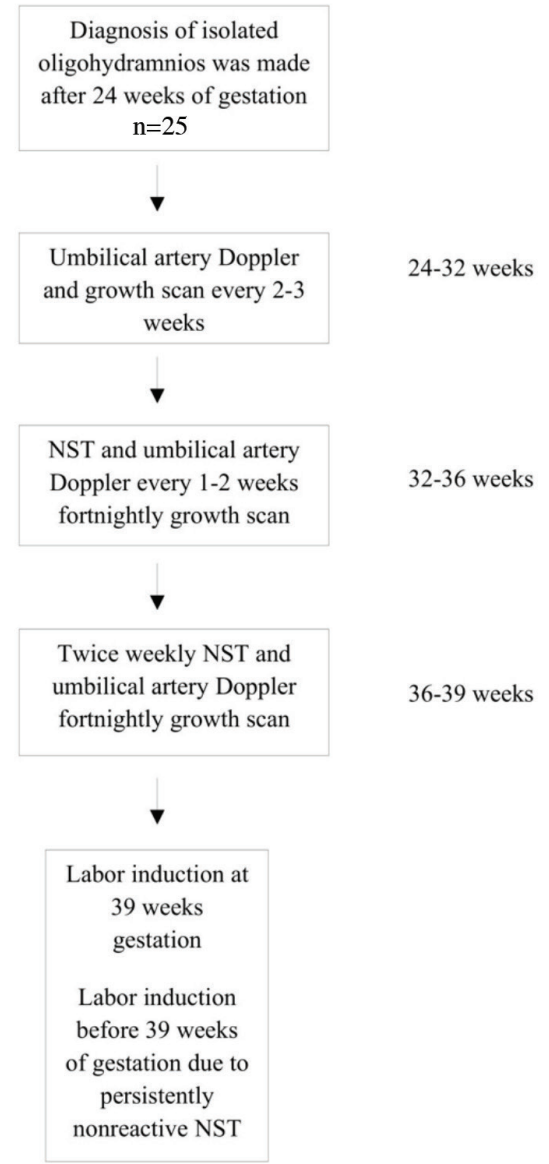

outcomes between the groups. Adverse perinatal outcomes were defined as: umbilical cord arterial $\mathrm{pH}<7.2$, Apgar 5 min $<7$, cesarean delivery for non-reassuring fetal heart-rate testing, transient tachypnea of the newborn (TTN), respiratory distress syndrome (RDS), admission to the neonatal intensive care unit (NICU), hypoxic ischemic encephalopathy (HIE), and neonatal death.

\section{Statistical analysis}

Statistical analysis was made with SPSS version 20.0 (IBM Inc., Armonk, NY, USA). Categorical data were analysed using the chi square test or Fisher's exact test. Numerical variables were compared using Student's t-test or Mann-Whitney U test. Pearson's rank correlation was used to assess the relationship between MPI and adverse perinatal outcomes. A p-value $<0.05$ and $r>0.5$ were considered to be statistically significant.

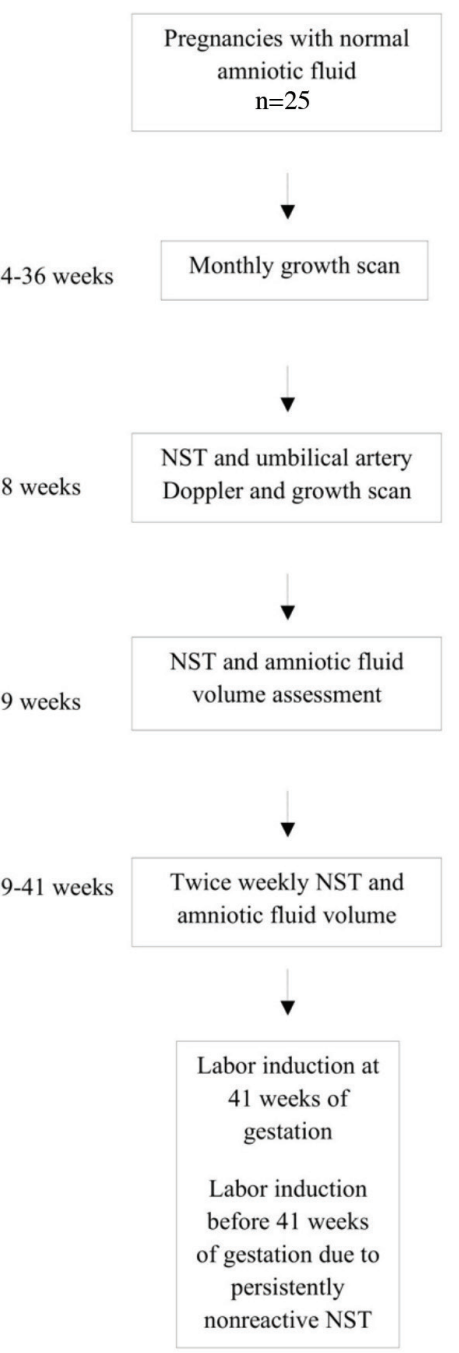




\section{Results}

The clinical characteristics of pregnacies with isolated oligohydramnios and normal amniotic fluid are illustrated in Table 1. Maternal age and nulliparity showed no difference. Gestational age at ultrasound and umbilical artery PI values were similar between the two groups. Unsurprisingly, mean AFI was significantly different at $31 \mathrm{~mm}$ and $109 \mathrm{~mm}$ in the isolated oligohydramnios and control group, respectively ( $\mathrm{p}<0.001)$.

Cardiac and renal artery Doppler parameters of pregnancies with isolated oligohydramnios and control group are presented in Table 2. Mean Mod-MPI value was significantly higher and IRT was longer in the isolated oligohydramnios group than the control group $(p=0.001$ and $p=0.009$, respectively). Mean ICT, ET values and mean renal artery PI value did not differ between the isolated oligohydramnios and control groups.

Perinatal outcomes in the isolated oligohydramnios and control group are presented in Table 3 . Gestational age at birth and birth weight were significantly lower $(p=0.041$ and $p=0.001$, respectively), and incidences of delivery before 37 weeks and cesarean section due to non-reassuring fetal heart rate testing were significantly more common $(p=0.034$ and $p=0.021$, respectively) in women with isolated oligohydramnios than the control group. Incidences of labor induction, arterial cord blood $\mathrm{pH}<7.2$, Apgar 5 min $<7$, meconium stained amniotic fluid, admission to NICU, TTN, HIE, RDS and neonatal death were not significantly different between the groups. Preterm induction of labor was performed in two patients with isolated oligohydramnios due to non-reassuring fetal heart rate at 36-37 weeks gestation. There was no significant correlation between Mod-MPI value and adverse perinatal outcomes (Pearson's $\mathrm{p}=0.112, \mathrm{p}=0.440)$.

\section{Discussion}

In the current study, it was observed that pregnancies with isolated oligohydramnios before 37 weeks were characterized by a higher rate of preterm delivery and a higher rate of cesarean delivery for non-reassuring fetal heart rate testing during labor. However, adverse perinatal outcomes such as arterial cord $\mathrm{pH}<7.2$, Apgar 5 min $<7$, meconium-stained amniotic fluid, admission to NICU and neonatal death were not significantly different between the pregnancies with isolated oligohydramnios and normal amniotic fluid volumes. Although we observed a higher rate of preterm delivery in the isolated oligohydramnios, the neonatal morbidity was similar. One possible explanation for this discrepancy might be the higher rate of late preterm deliveries at a gestational age of 36-37 weeks. Although it has been reported that isolated oligohydramnios at preterm pregnancy is associated with higher rate of adverse neonatal outcomes, subgroup analysis showed that adverse outcomes were substantially related to the induction of delivery but not to the isolated oligohydramnios itself (6). Melamed et al. (6) showed that the neonatal outcome in the isolated oligohydramnios group (expectant-management) was similar to that observed in the control group.

Table 1. The clinical characteristics of pregnancies with isolated oligohydramnios and control group

\begin{tabular}{|l|l|l|l|}
\hline & Isolated oligohydramnios & Control group & p \\
\hline $\mathrm{N}$ & 25 & 25 & - \\
\hline Maternal age (years) & $30.6 \pm 6.3$ & $29.8 \pm 5.4$ & 0.668 \\
\hline Nulliparity & $14(56)$ & $12(48)$ & 0.778 \\
\hline Gestational age at ultrasound (weeks) & $30.2 \pm 4.8$ & $30 \pm 5$ & 0.83 \\
\hline Mean AFI (mm) & $31(21-46)$ & $109(90-140)$ & $<0.001$ \\
\hline Umbilical artery PI & $0.99 \pm 0.14$ & $0.99 \pm 0.17$ & 0.907 \\
\hline Data are presented as mean \pm standard deviation or n (\%), AFI: Amniotic fluid index, PI: Pulsatility index \\
\hline
\end{tabular}

Table 2. Cardiac and renal artery Doppler parameters of pregnancies with isolated oligohydramnios and control group

\begin{tabular}{|l|l|l|l|}
\hline & Isolated oligohydramnios & Control group & p \\
\hline $\mathrm{N}$ & 25 & 25 & - \\
\hline Modified myocardial performance index & $0.53 \pm 0.11$ & $0.43 \pm 0.08$ & 0.001 \\
\hline Isovolumetric contraction time (ms) & $36.6 \pm 0.9$ & $32.1 \pm 0.7$ & 0.069 \\
\hline Ejection time (ms) & $161.7 \pm 19.4$ & $169.8 \pm 10.9$ & 0.074 \\
\hline Isovolumetric relaxation time (ms) & $48.6 \pm 1.0$ & $41.2 \pm 0.9$ & 0.009 \\
\hline Mean renal artery PI & $2.31 \pm 0.41$ & $2.31 \pm 0.23$ & 0.995 \\
\hline Data are presented as mean \pm standard deviation, PI: Pulsatility index & \\
\hline
\end{tabular}


Table 3. Perinatal outcomes in the isolated oligohydramnios and control group

\begin{tabular}{|l|l|l|l|}
\hline & Isolated oligohydramnios & Control group & p \\
\hline $\mathrm{N}$ & 25 & 25 & - \\
\hline Gestational age at birth (weeks) & $36.9 \pm 2.7$ & $38.4 \pm 2.1$ & 0.041 \\
\hline$<37$ weeks & $8(32)$ & $2(8)$ & 0.034 \\
\hline$<34$ weeks & $2(8)$ & $1(4)$ & 0.552 \\
\hline Birth weight (g) & $2660 \pm 570$ & $3199 \pm 464$ & 0.001 \\
\hline Labor induction & $6(24)$ & $2(8)$ & 0.123 \\
\hline Cesarean delivery & $14(56)$ & $7(28)$ & 0.045 \\
\hline Non-reassuring fetal heart rate & $7(28)$ & $1(4)$ & 0.021 \\
\hline Meconium-stained amniotic fuid & 0 & 0 & N/A \\
\hline Arterial cord pH $<7.2$ & 0 & 0 & N/A \\
\hline 5 -min Apgar score $<7$ & $3(12)$ & 0 & 0.07 \\
\hline Neonatal intensive care unit admission & $4(16)$ & $1(4)$ & 0.157 \\
\hline Transient tachypnea of the newborn & 2 & 0 & 0.149 \\
\hline Respiratory distress syndrome & 2 & 1 & 0.552 \\
\hline Hypoxic ischemic encephalopathy & 0 & 0 & N/A \\
\hline Neonatal death & 0 & 0 & N/A \\
\hline Data are presented as mean \pm standard deviation or n (\%), N/A: Not applicable & & \\
\hline
\end{tabular}

A recent meta-analysis including six studies of isolated oligohydramnios in 27,526 women showed that pregnancies with isolated oligohydramnios had significantly higher rates of meconium aspiration syndrome, cesarean delivery for fetal distress and admission to the NICU. It was concluded that isolated oligohydramnios is a pathological finding and there are not sufficient data to determine the optimal timing of delivery to reduce the risk of adverse outcomes (5). Several other studies have reported that perinatal outcomes in pregnancies with isolated oligohydramnios were similar to pregnancies with normal amniotic fluid $(17,18)$. Our findings support the studies reporting that isolated oligohydramnios is not significantly associated with adverse perinatal outcome $(6,17,18)$.

The amount of amniotic fluid depends on renal filtration and urine production. Renal artery Doppler velocimetric parameters reflect the arterial perfusion of the kidneys and these parameters may be related to amniotic fluid level. Several studies investigated the relationship between fetal renal artery Doppler parameters and amniotic fluid level but the results of these studies were inconsistent $(14,15,19,20)$. Yoshimura et al. (20) showed that renal artery PI is significantly higher in pregnancies with isolated oligohydramnios compared to pregnancies with normal amniotic fluid. In another study, including 63 pregnancies followed from 16 to 41 weeks, there was no correlation between amniotic fluid levels and fetal renal artery PI (14). Benzer et al. (15) evaluated renal artery PI in pregnancies with oligohydramnios at 22, 28 and 34 weeks of gestation and found significantly higher renal artery PI only at 28 weeks of gestation, but not at 22 or 34 weeks. Budunoglu et al. (16) reported that renal artery PI was not significantly different between patients with isolated oligohydramnios and normal amniotic fluid at 25 to 40 weeks of gestation. In the current study, renal artery PI was not significantly different between pregnancies before 37 weeks of gestation with isolated oligohydramnios and normal amniotic fluid, suggesting that isolated oligohydramnios may not be related to impaired renal artery blood flow.

Mod-MPI values are associated with fetal left ventricular function and it has become a reliable marker of fetal cardiac dysfunction. Fetal MPI has been evaluated in several high-risk pregnancies, such as diabetic and postterm pregnancies, fetal growth restriction and twintwin transfusion syndrome $(12,21,22)$. We evaluated ModMPI in pregnancies with isolated oligohydramnios and demonstrated subtle cardiac dysfunction in fetuses with isolated oligohydramnios compared with healthy controls. The higher Mod-MPI levels have been found to be primarily due to an elevated IRT. IRT becomes abnormal in the initial stages of cardiac dysfunction and is mainly caused by decreased diastolic compliance (23). Calcium reuptake of myocardial cells are reduced, which leads to prolongation of complete cardiomyocyte relaxation and an increased IRT (24). Based on the findings of the present study, we speculate that there maybea mild diastolic dysfunctioninfetuses with isolated oligohydramnios. However, whether oligohydramnios is the cause per se or the consequence of mild diastolic dysfunction 
is not clear. There is only one study that has evaluated the association between an increased volume of amniotic fluid and Mod-MPI in pregnancies with isolated polyhydramnios. In that study, Mod-MPI was significantly higher in isolated polyhydramnios compared with controls and Mod-MPI was also associated with adverse perinatal outcome (25). However, although Mod-MPI was significantly increased in isolated oligihydramnios pregnancies in our study, we could not demonstrate any association between Mod-MPI values and adverse perinatal outcomes in fetuses with isolated oligohydramnios. Our study suggests that Mod-MPI evaluation does not seem to help in the management of pregnancies before 37 weeks of gestation with isolated oligohydramnios.

The strength of this study was its prospective design and measurement of Doppler parameters by a single experienced examiner. However, the limitation of the study was the small sample size.

\section{Conclusion}

Fetuses with isolated oligohydramnios have increased Mod-MPI which may be due to mild fetal cardiac diastolic dysfunction. Increased Mod-MPI was not associated with adverse perinatal outcomes in fetuses with isolated oligohydramnios. Further large studies are needed to investigate theimportance of Mod-MPI in isolated oligohydramnios.

Ethics Committee Approval: The study was approved by the Ethical Committee of the Istanbul University-Cerrahpaşa, Cerrahpaşa Faculty of Medicine (approval number: 215728).

\section{Informed Consent: It was obtained.}

\section{Peer-review: Externally peer-reviewed.}

Author Contributions: Surgical and Medical Practices: R.M., H.E., A.Ö., F.Ö.; Concept: R.M., H.E., A.Ö., F.Ö.; Design: R.M., H.E., A.Ö., F.Ö.; Data Collection or Processing: R.M., H.E., A.Ö., F.Ö.; Analysis or Interpretation: R.M., H.E., A.Ö., F.Ö.; Literature Search: R.M., H.E., A.Ö., F.Ö.; Writing: R.M., H.E., A.Ö., F.Ö.

Conflict of Interest: No conflict of interest is declared by the authors.

Financial Disclosure: The authors declared that this study received no financial support.

\section{References}

1. Owen J, Albert PS, Buck Louis GMB, Fuchs KM, Grobman WA, Kim $\mathrm{S}$, et al. A contemporary amniotic fluid volume chart for the United States: The NICHD Fetal Growth Studies-Singletons. Am J Obstet Gynecol 2019; 221: 67.e1-67.e12.
2. Rosati P, Guariglia L, Cavaliere AF, Ciliberti P, Buongiorno S, Ciardulli A, et al. A comparison between amniotic fluid index and the single deepest vertical pocket technique in predicting adverse outcome in prolonged pregnancy. J Prenat Med 2015; 9: 12-5.

3. Rossi AC, Prefumo F. Perinatal outcomes of isolated oligohydramnios at term and post-term pregnancy: a systematic review of literature with meta-analysis. Eur J Obstet Gynecol Reprod Biol 2013; 169: 149-54.

4. Shrem G, Nagawkar SS, Hallak M, Walfisch A. Isolated oligohydramnios at term as an indication for labor induction: a systematic review and meta-analysis. Fetal Diagn Ther 2016; 40: 161-73.

5. Rabie N, Magann E, Steelman S, Ounpraseuth S. Oligohydramnios in complicated and uncomplicated pregnancy: a systematic review and meta-analysis. Ultrasound Obstet Gynecol 2017; 49: 442-9.

6. Melamed N, Pardo J, Milstein R, Chen R, Hod M, Yogev Y. Perinatal outcome in pregnancies complicated by isolated oligohydramnios diagnosed before 37 weeks of gestation. Am J Obstet Gynecol 2011; 205: 241.e1-6.

7. Friedman D, Buyon J, Kim M, Glickstein J. Fetal cardiac function assessed by Doppler myocardial performance index (Tei Index). Ultrasound Obstet Gynecol 2003; 21: 33-6.

8. Hernandez-Andrade E, López-Tenorio J, Figueroa-Diesel $\mathrm{H}$, Sanin-Blair J, Carreras E, Cabero L, et al. A modified myocardial performance (Tei) index based on the use of valve clicks improves reproducibility of fetal left cardiac function assessment. Ultrasound Obstet Gynecol 2005; 26: 227-32.

9. Hernandez-Andrade E, Benavides-Serralde JA, Cruz-Martinez R, Welsh A, Mancilla-Ramirez J. Evaluation of conventional Doppler fetal cardiac function parameters: E/A ratios, outflow tracts, and myocardial performance index. Fetal Diagn Ther 2012; 32: 22-9.

10. Bhorat I, Bagratee J, Pillay M, Reddy T. Determination of the myocardial performance index in deteriorating grades of intrauterine growth restriction and its link to adverse outcomes. Prenat Diagn 2015; 35: 266-73.

11. Sanhal CY, Daglar HK, Kara O, Uygur D, Yucel A. Assessment of fetal myocardial performance index in women with pregestational and gestational diabetes mellitus. J Obstet Gynaecol Res 2017; 43: 65-72.

12. Ozel A, Alici Davutoglu E, Yildirim S, Madazli R. Fetal cerebral and cardiac hemodynamics in postdate pregnancy. J Matern Fetal Neonatal Med 2019; 32: 3458-63.

13. Verburg BO, Geelhoed J, Steegers E, Hofman A, Moll HA, Witteman JC, et al. Fetal kidney volume and its association with growth and blood flow in fetal life: The Generation R Study. Kidney Int 2007; 72: 754-61.

14. Figueira CO, Surita FG, Dertkigil MS, Pereira SL, Bennini JR Jr, Morais SS, et al. Longitudinal reference intervals for Doppler velocimetric parameters of the fetal renal artery correlated with amniotic fluid index among low-risk pregnancies. Int J Gynaecol Obstet 2015; 131 : 45-8.

15. Benzer N, Pekin A, Yılmaz SA, Kerimoğlu ÖS, Doğan NU, Çelik Ç. Predictive value of second and third trimester fetal renal artery Doppler indices in idiopathic oligohydramnios and polyhydramnios in low-risk pregnancies: a longitudinal study. J Obstet Gynaecol Res 2015; 41: 523-8.

16. Budunoglu MD, Yapca OE, Yldildiz GA, Atakan Al R. Fetal renal blood flow velocimetry and cerebro-placental ratio in patients with isolated oligohydramnios. J Gynecol Obstet Hum Reprod 2019; 48: 495-9.

17. Zhang J, Troendle J, Meikle S, Klebanoff MA, Rayburn WF. Isolated oligohydramnios is not associated with adverse perinatal outcomes. BJOG 2004; 111: 220-5.

18. Manzanares S, Carrillo MP, González-Perán E, Puertas A, Montoya F. Isolated oligohydramnios in term pregnancy as an indication for induction of labor. J Matern Fetal Neonatal Med 2007; 20: 221-4. 
19. Mari G, Kirshon B, Abuhamad A. Fetal renal artery flow velocity waveforms in normal pregnancies and pregnancies complicated by polyhydramnios and oligohydramnios. Obstet Gynecol 1993; 81: 560-4.

20. Yoshimura S, Masuzaki H, Gotoh H, Ishimaru T. Fetal redistribution of blood flow and amniotic fluid volume in growth-retarded fetuses. Early Hum Dev 1997; 47: 297-304.

21. Ichizuka K, Matsuoka R, Hasegawa J, Shirato N, Jimbo M, Otsuki K et al. The Tei index for evaluation of fetal myocardial performance in sick fetuses. Early Hum Dev 2005; 81: 273-9.

22. Alici Davutoglu E, Ozel A, Oztunc F, Madazli R. Modified myocardial performance index and its prognostic significance for adverse perinatal outcome in early and late onset fetal growth restriction. $\mathrm{J}$ Matern Fetal Neonatal Med 2020; 33: 277-82
23. Mahajan A, Henry A, Meriki N, Hernandez-Andrade E, Crispi F, Wu $\mathrm{L}$, et al. The (Pulsed-Wave) Doppler fetal myocardial performance index: technical challenges, clinical applications and future research. Fetal Diagn Ther 2015; 38: 1-13.

24. Crispi F, Gratacós E. Fetal cardiac function: technical considerations and potential research and clinical applications. Fetal Diagn Ther 2012; 32: 47-64.

25. Gezer C, Ekin A, Ozeren M, Taner CE, Mat E, Solmaz U. Can the myocardial performance index be used as a new predictive factor for a poor prognosis in fetuses with idiopathic polyhydramnios? J Ultrasound Med 2016; 35: 2649-57. 